\title{
SIMPLE PROCEDURE FOR SUPERCONDUCTING COIL WINDING
}

\author{
A. Mikhailichenko, T. Moore \\ Cornell University, Laboratory of Nuclear Studies, Ithaca, NY 14853
}

\begin{abstract}
The hardware and non-toxic procedure for winding of racetrack type superconducting single layer coils described. A 43-turn coil winding takes about 5 minutes. The coil dimensions remaining within $\pm 0.05-\mathrm{mm}$ margins after the impregnation is cured.
\end{abstract}

\section{INTRODUCTION}

This work was initiated in a framework of the possibility to upgrade Cornell storage ring CESR with dual bore focusing magnets described in [1]. These magnets must be able to accommodate two independent vacuum chambers. Fully completed unit, sharing the same cryostat, will include dual bore quadrupole, dual bore sextupole, and dual bore dipole correctors [1-3]. Some magnets might have skew quadruples or octupoles instead of dipole correctors. It was suggested there, that existing CESR magnets could be modified with some magnetic lips allowing extended good field region. The first prototype of dual bore quadrupole lens was tested in Dewar [2]. The test showed that the basic design satisfies requirements in principle, however some improvements still needed to be done. The latest results of this modified magnet test were represented in [4]. These measurements in a Dewar indicated satisfactory field quality.

In [5] the present situation with dual bore quadrupole and sextupole is summarized.

In this report we describe the technology of fabrication of thin racetrack-type coils, which might be useful in the future, independently on any specific project.

\section{THE MAGNET}

The design of dual bore magnet is described in $[2,5,6]$. Each of the quadrupoles is a combination of two transversely separated quadrupole magnets that share the same iron yoke. The same is valid for sextupole. Separation of axes needs to be as small as possible to reduce the size of the room temperature dipole magnet. Technical limitations make this distance about $80 \mathrm{~mm}$. So in design accepted, the field is formed not only by the poles but also in the same amount by the coils shape and their positioning. This type of lens was suggested in [4]. The field quality required for the quadrupole is $\left[B_{y}(x)-G \cdot x\right] / G \cdot x \leq 5 \cdot 10^{-4}$, where $B_{y}(x)$ is the field across the aperture, $G$ is a gradient. That is why the tolerances of coil dimension are high. Calculations show that the accuracy of pole fabrication must be of the order of $\delta \approx 8.5 \cdot 10^{-3} \mathrm{~mm}$. The accuracy of the coil winding must remain within \pm 0.05 -mm margins. That was obtained using numerical modeling. Basically this is a fraction of the wire diameter, which is $\cong 0.43 \mathrm{~mm}$. The designed currents were about $100 \mathrm{~A}$ and $35 \mathrm{~A}$ for quadrupole and sextupole respectively.

In the presence of neighboring lens, magnetic lines lose its quadrupole symmetry in the yoke, and, hence in each aperture. Now not only quadrupole-associated harmonics are allowed. Dipole and sextupole components are the strongest among allowed now. The same happens, sequentially, for the whole lens. Proper modeling includes the lowest symmetry piece taking into account this effect [6]. To minimize this cross-influence of the lenses the poles need to be made slightly different. The coils remain identical, however. For reduction of cross interference of the field at the fringe, special 1-mm thick iron covers cover the ends of the coils.

For the requirements of the beam optics the gradients in both neighboring lenses have the same sign and about the same value.

The superconducting coils used there have straight sections inside the body of lens and turning around at the ends. The coils are a single layer. So basically the field formation by the coils is the class of a current sheet. One can also consider this type of lens as Panofsky-Hand type but with hyperbolic poles at the corners.

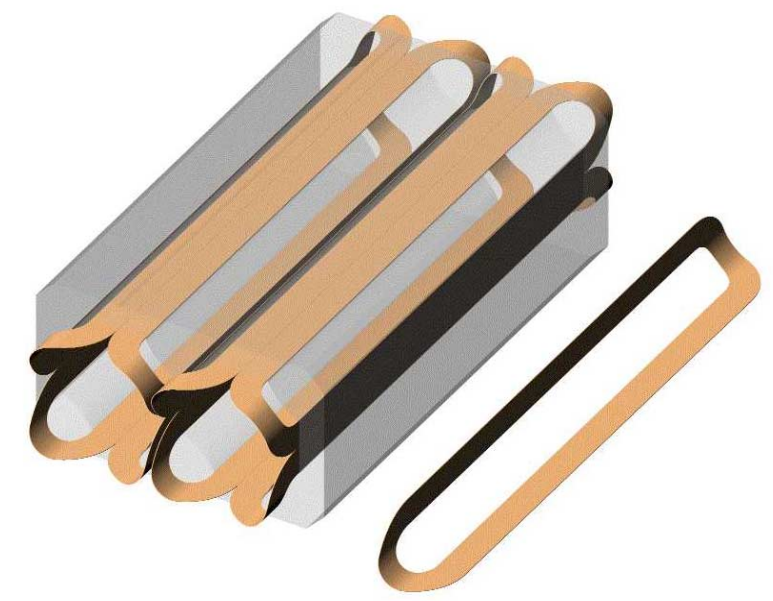

Figure 1: Windings are single layer racetrack coils, bent on 90 degrees along short side with radius. They occupy the whole straight sides of the yoke. Yoke septum between the apertures is $4 \mathrm{~mm}$. The magnet yoke is shown semi-transparent. One coil represented at the right separately.

For sextupole this requirement is ten times weaker. However technology used makes the sextupole coils the same accuracy. 


\section{THE CONCEPT}

As one can see, the coils itself are the saddle type ones. We wound the coils flat however, as we found that the coils could be bent along the short side without any damage. So we wound coils flat and bent after, so basically we came to a technology for flat winding. The bending was done with simple two-plate device. As the yoke ends before the region of the bend, influence of the exact radius of the bend is minimal.

As positioning of the wires is not possible during the winding itself, we decided position of the wires more accurately during the curing process. In other words the constant pressure applied during the curing process squeezed the wires together. Making both sides of the winding fixture movable does the squeezing. The winding fixture is used during the curing process.

The quadrupole coil dimensions defined by the size of the magnet has the outer size of $458.5 \mathrm{~mm}$, the width of the racetrack is 18.68 , the outer width is $72.12 \mathrm{~mm}$, thickness of $0.43 \mathrm{~mm}$.

We tested a wire with a few types of insulation and coating. First was a bare diameter of $0.017^{\prime \prime}$ wire with Formvar insulation.

As it turned out, we ended our search after the wire from former SSC correcting coils with Kapton insulation and Bondall impregnation came into our disposal.

For the wire with Formvar insulation we tested a few epoxy compounds. Among them were EPO-TEK 905, Epoxy Technology; ECCOBOUND, Resin 285, Black with Catalyst 24LV, W.R. Grace\&Com.. This product is now available as STYCAST 2850FT from Emerson\&Cuming.

\section{WINDING FIXTURE}

The coil is wound between two plates having the gap very close to the wire diameter. Special screws align the plates so that the gap is even all around. The wire goes in the gap with friction.

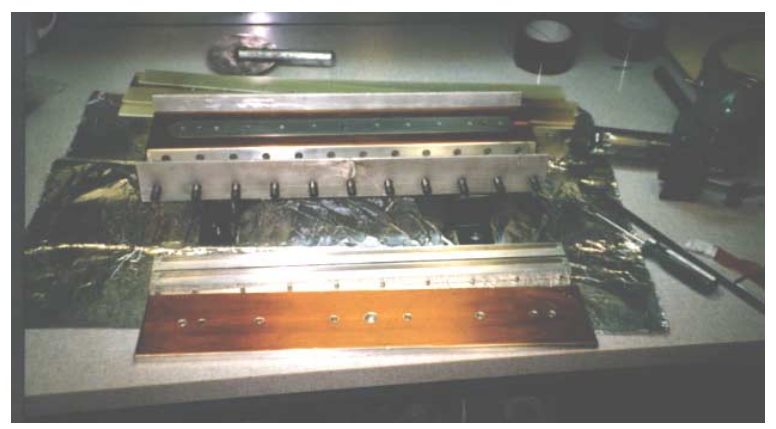

Figure 2: The winding set. At the top of the picture the pizza cutter style roller is visible.

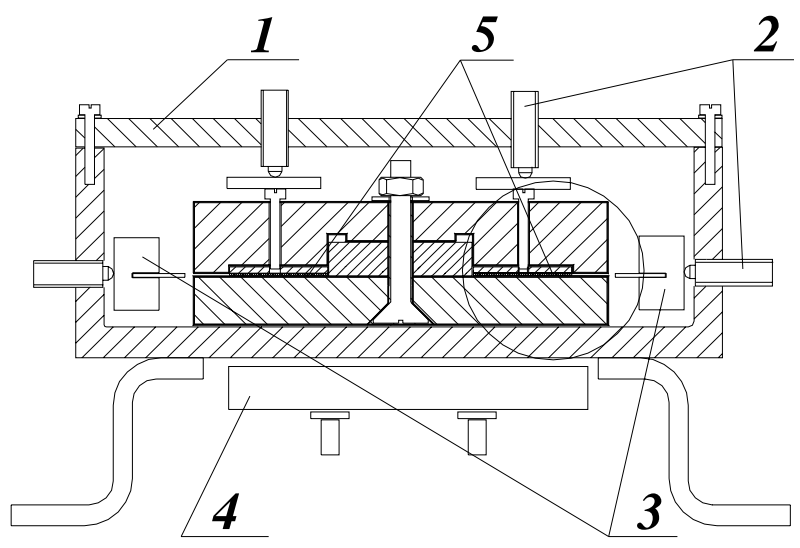

Figure 3: Winding fixture itself is shown here inside the curing cabinet, equipped with springing bolts. These bolts push the walls to proper positions. 1-cabinet, 2springing bolts. The side knife-like walls 3 are shown in "out" position. 4- heater, 5-the coil.

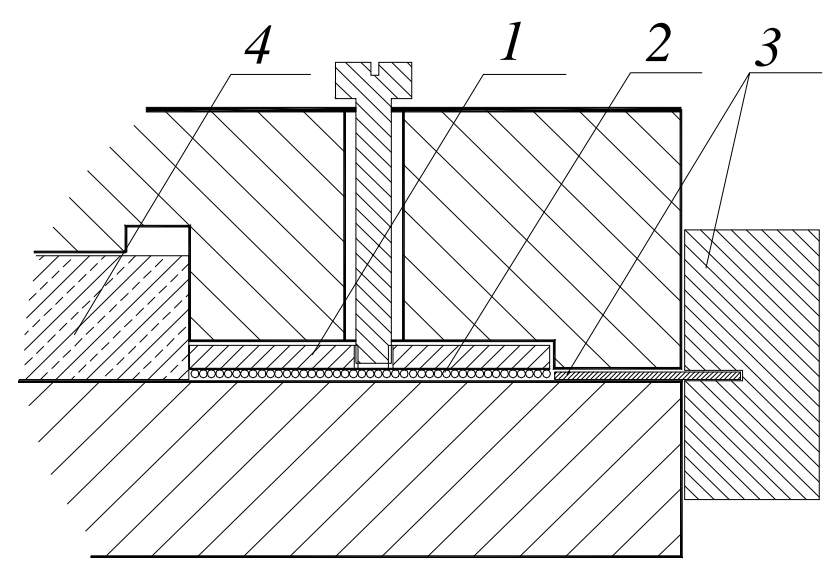

Figure 4: Scaled view from Fig.3, circled. 1-the inner side plate, pressed by bolts; in it's turn bolts are pressed by additional spring loaded setscrews, marked in Fig. 3 as 2, 2-the coil, 3-the knife edge with its holder, 4-is a central island.

When the holder of the knife touches the wall, the depth is exactly the same as required for the proper width of the coil. So basically the mechanical accuracy of the fabrication defines the accuracy of the wire positioning. The winding fixture has dowel pins for accurate positioning of the parts.

Epoxy was introduced during the winding, passing the wire through a small hole in the plastic cup

With SSC wire the amount of Bondall coating $\cong 0.005^{\prime \prime}$ was about right to fill the gaps between the wires. The Kapton itself, the same thickness, also deformed during the pressure application allows the freedom to move for the wires. 


\section{DESCRIPTION OF THE PROCEDURE}

For preliminary compressing the wires and pushing them into the slit during the winding, a special roller, looking pretty mach the same as a pizza cutter (except the sharpen edges), was used successfully.

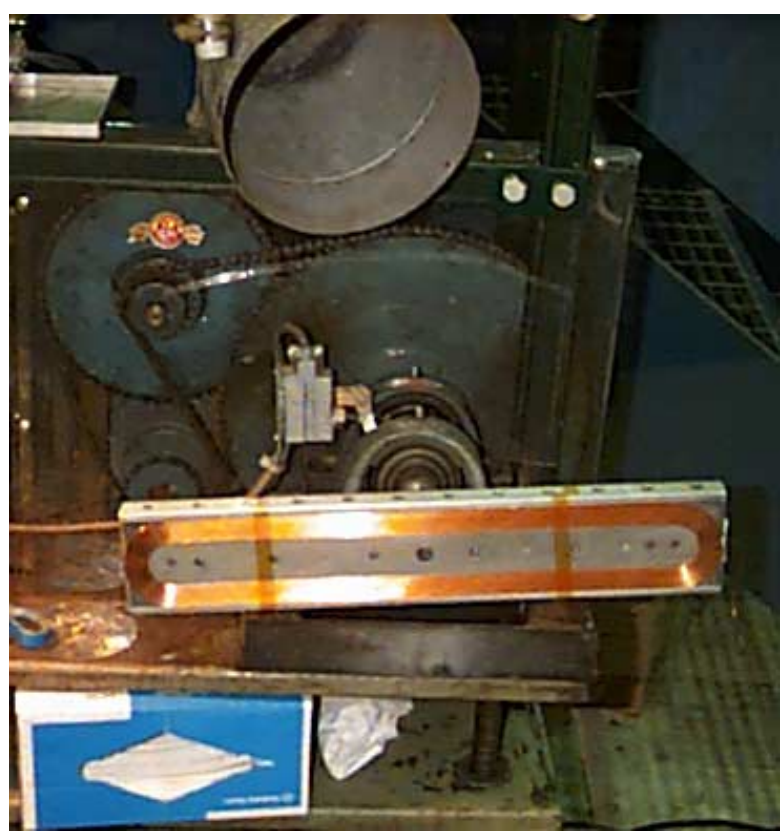

Figure 5: The winding fixture installed in the winding machine shown. The top cover removed, so the coil could be visible. SC wire is coming vertically from the spool located at the top of machine. Tension of the wire regulated with special brake.

As it was mentioned, after the winding completed the epoxy allowed curing in the fixture. The last one was positioned for this purpose into cabinet, having the springing bolts along the walls. When the Bondall coated wire used in a latest development, an electrical heater heats the cabinet.

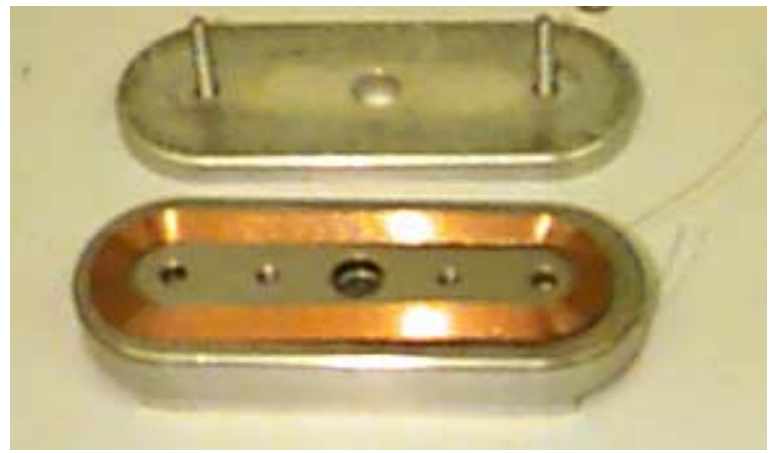

Figure 6: The winding fixture for sextupole coil. Basically it is a scaled version of he fixture used for quadrupole coil winding.

To prevent the parts from sticking together standard Teflon spray was used successfully.
The sextupole coil has the big outer size of $133 \mathrm{~mm}$, the width of the racetrack is $10.35 \mathrm{~mm}$, the outer width is 42 $\mathrm{mm}$, thickness of the coil is $0.45 \mathrm{~mm}$, number of turns is23.

As it was mentioned the best performance gave the wire Wrapped by Kapton and impregnated by Bondall. In that case the winding becomes much cleaner and the fumes were gone. After winding, we just heated the coil remaining in the fixture up to $260 \mathrm{~F}$.

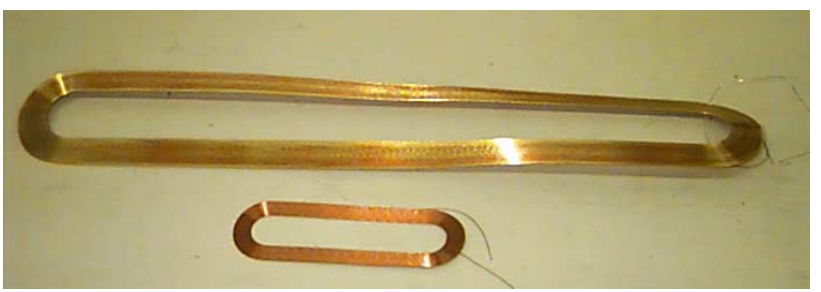

Figure 7: The coils, removed from the fixture. The coils are flexible along the wire plane, but keep the transverse dimensions. After positioning into the magnet, the coils become well attached to the surface of the iron.

\section{CONCLUSION}

Technology developed allow fast and accurate winding of a racetrack type coils. Two different coil sizes were used with this technology successfully. Utilization of Bondall (and latest replacements for it, such as $3 \mathrm{M}$ compound) type pre-coating for the wire with Kapton insulation showed the best performance for the coils.

We represented these materials, as they might be useful for any other project with superconducting coils as well.

This work supported by National Science Foundation.

\section{REFERENCES}

[1] D. Rubin, M. Tigner, Shared bends and Independent Quadrupoles, Cornell CON 94-28, 1994.

[2] A. Mikhailichenko, D. Rubin, Concentric Ring Colliding Beam Machine with Dual Aperture Quadrupoles, Cornell CLNS 96-1420, 1996.

[3] G. Dugan, A.A.Mikhailichenko, J. Rojers, D. Rubin, Dual Aperture High Luminosity Collider at Cornell, Talk on Particle Accelerator Conference PAC 97, Vancouver, B.C. Canada, 12-17 May 1997, 6B10. Proceedings, p. 318.

[4] A.A. Mikhailichenko, Some features of superconducting dual bore Lens, CLNS99/1609, and Presented on PAC99.

[5] A. Mikhailichenko, Quadrupole and Sextupole for Dual Bore Ring, RPPH321, this conference.

[6] A. Mikhailichenko, 3D Electromagnetic Field. Representation and measurement, CBN 95-16, Cornell, 1995. 